\title{
NEON: Near-Accurate Efficient FIR Filter for Ultra Low-Power Applications
}

\author{
Srimai Inapurapu, JVR Ravindra \\ Center for Advanced Computing Research Laboratory (C-ACRL) \\ Department of Electronics and Communication Engineering \\ Vardhaman College of Engineering, Shamshabad, Kacharam \\ Hyderabad, Telangana, India \\ email: srimai.inapurapu@gmail.com, jayanthi@ieee.org
}

\begin{abstract}
Low-power dissipation is an imperative requirement in the design of an efficient Digital Signal Processing system which is employed in many multimedia applications such as image and video processing. Finite Impulse Response (FIR) filter is indispensable in the design of several such Digital Signal Processing (DSP) applications. The output of these applications, either an image or a video can be nearly accurate for human perception. This toleration in the loss of quality of the output can be exploited to design an energy-efficient system by using approximate computation. Moreover, the efficacy of a system can be improved multi-fold by using reversible logic which benefits in the design of ultra-low-power systems. In this paper, we propose an approximate adder using a reversible Toffoli gate and employ it in designing NEON (Near-accurate and Efficient FIR filter for ultra low-power applicatiONs). Simulation results carried out using Cadence (c) design tools in $45 \mathrm{~nm}$ technology node show that the FIR Filter designed using the proposed adder gives significantly better results compared to the designs using the adders in literature. Experiment results using ISCAS benchmarks and comparison with previous methods demonstrate the effectiveness of the proposed method. In addition to producing fewer garbage outputs, the FIR filter designed using the proposed adder yields power reduction of $74 \%$, delay reduction of $64 \%$ and PowerDelay Product reduction of $\mathbf{9 0 . 1 \%}$.
\end{abstract}

\section{INTRODUCTION}

Typically used multimedia applications involving image or video processing have Digital Signal Processing blocks as the backbone. Finite Impulse Response (FIR) filters are widely used in these applications. The resultant output, usually an image or a video, can be nearly accurate for human perception. This relaxation on producing strictly accurate outputs facilitates in carrying out approximate computation. This flexibility can be utilized in designing low-power systems.

Earlier works targeting design of low-power systems using proximate computations include Algorithmic Noise Tolerance (ANT) [1-4] which focus on limiting the errors by using the concept of Voltage OverScaling. A low-power approximate adder is proposed in [5], which functions by separating the inputs into accurate and approximate parts. Probabilistic Computing has been proposed as an alternative in [6-8] which produces inexact outputs in order to achieve less power consumption and hardware complexity. Various inaccurate adders have been proposed in [9-10] which embrace error to achieve reduced power dissipation.
An imprecise specification has been proposed in [11] which focuses on improving RTL specification by adding new semantic elements. Woo[12] has proposed an analog adder using approximate computation.

Of late, reversible logic has emerged as a paradigm in designing energy-efficient systems. Landauer [13] has proved that for irreversible logic computations, every bit of information lost results in the generation of KT $\log 2$ joules of heat energy. Bennet [14] showed that this heat dissipation would not occur in reversible computation. Several reversible gates have been proposed such as Fredkin Gate [15], Toffoli Gate [16] and Feynman Gate [17-18]. Low-power adder circuits are designed using a new reversible gate proposed in [19]. Using reversible gates, several adders and multipliers are designed in [20-30] which dissipate low-power. An FIR filter model is designed using reversible logic gates in [33]. In [34], an ultra low-power FIR filter is proposed using subthreshold design approach. One of the important concerns in designing a reversible logic system is to use as few reversible logic gates as possible and produce fewer number of garbage outputs.

FIR filters use several addition operations. To design an energy-efficient low-power FIR filter, it is imperative to design low-power adder cells. Our approach is described as follows: First, an approximate full adder cell is designed using reversible logic. This adder cell is later utilized in the design of an FIR filter (NEON).

Despite the emergence of several approximation algorithms, limited amount of work has been focused on approximating FIR Filters. Previous work [33] presents the design of FIR Filter using reversible Fredkin gates. However, this design produces many garbage outputs and consumes a lot of power and thereby presents hindrance to the design of an efficient Digital Signal Processing system. To the best of our knowledge, this work presents the first approximate reversible adder which not only produces minimal garbage outputs but also yields significant reductions in terms of power, delay and Power-Delay Product.

The remainder of the paper is organized as follows. Section II outlines previous work related to the emergence of Approximation Algorithms and Reversible Logic. In section III, an approximate full adder using reversible logic gates is presented. In section IV, we propose NEON. In section $\mathrm{V}$, we compare NEON with the design of FIR filters using existing adders 
in terms of power, delay and Power Delay Product (PDP). Section VI concludes the paper.

\section{PREVIOUS WORK}

Approximate Computing has emerged as a paradigm shifting technique in the design of ultra lowpower systems whose applications can tolerate error. Of all the techniques proposed previously, we make use of Logic Complexity Reduction technique which minimizes the Boolean Expressions [9-11] resulting in reduced hardware overhead and thereby CPU time.

\section{A. Reversible Logic}

Reversible Logic bestows in carrying out lowpower computations. Irreversible logic computations result in the dissipation of KT $\log 2$ joules of heat energy for every bit of information lost. In reversible systems, the aforementioned heat dissipation would not occur as the system's input vectors can be retrieved from output vectors. There is a one-to-one correspondence between input and output assignments [13-14] in reversible systems. Hence, an input vector (I) can be uniquely obtained from the output vector $(\mathrm{O})$ in a reversible system. Block diagram of a reversible system is shown in Fig. 1. below.

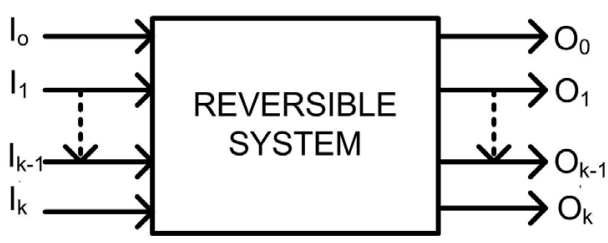

Fig. 1. Reversible System

A $k \times k$ reversible gate can be represented as:

$$
\begin{gathered}
I=\left(I_{0}, I_{1} \ldots \ldots I_{k-1}, I_{k}\right) \\
O=\left(O_{0}, O_{1} \ldots \ldots O_{k-1}, O_{k}\right)
\end{gathered}
$$

We propose the design of approximate Full-Adder cell using a reversible Toffoli gate.

\section{B. Toffoli Gate}

Toffoli Gate is a 3 X 3 Reversible Gate with input and output equations as follows [16]:

$$
\begin{gathered}
I=(A, B, C) \\
O=(A, B, A B \oplus C)
\end{gathered}
$$
Fig.2.

The block diagram of Toffoli Gate is shown in

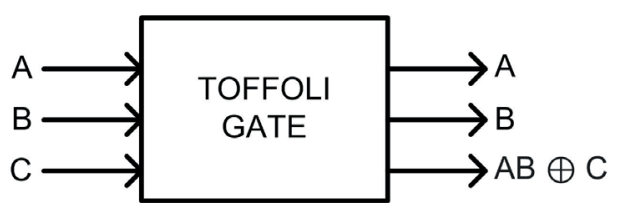

Fig. 2. Toffoli Gate

\section{Novel Proposed Approximate AdDER}

In this section, the design of a low-power Approximate Full-Adder cell is discussed. A close examination of the truth table of a conventional Full Adder shows that $\mathrm{Sum}=\mathrm{B}$ and $\mathrm{C}_{\text {out }}=\mathrm{A}$ for 4 cases out of 8. As shown in Table $\mathrm{I}$, for the input combinations $\mathrm{A}$ $=0, \mathrm{~B}=0, \mathrm{C}_{i n}=0 ; \mathrm{A}=0, \mathrm{~B}=1, \mathrm{C}_{i n}=0 ; \mathrm{A}=$ $1, \mathrm{~B}=0, \mathrm{C}_{i n}=1$ and $\mathrm{A}=1, \mathrm{~B}=1, \mathrm{C}_{i n}=1$, the approximated Full Adder's output is similar to that of a conventional Full Adder.

TABLE I. TRUTH TABLE FOR CONVENTIONAL FUll AdDER AND APPROXIMATED ADDER

\begin{tabular}{|c|c|c|c|c|c|c|}
\hline \multicolumn{3}{|c|}{ Inputs } & \multicolumn{2}{c|}{ Accurate Outputs } & \multicolumn{2}{c|}{ Approximate Outputs } \\
\hline $\mathrm{A}$ & $\mathrm{B}$ & $\mathrm{C}_{\text {in }}$ & Sum & $\mathrm{C}_{\text {out }}$ & Sum & $C_{\text {outa }}$ \\
\hline 0 & 0 & 0 & 0 & 0 & $0 \sqrt{ }$ & $0 \sqrt{ }$ \\
\hline 0 & 0 & 1 & 1 & 0 & $0 \times$ & $0 \sqrt{ }$ \\
\hline 0 & 1 & 0 & 1 & 0 & $1 \sqrt{ }$ & $0 \sqrt{ }$ \\
\hline 0 & 1 & 1 & 0 & 1 & $1 \times$ & $0 \times$ \\
\hline 1 & 0 & 0 & 1 & 0 & $0 \times$ & $1 \times$ \\
\hline 1 & 0 & 1 & 0 & 1 & $0 \sqrt{ }$ & $1 \sqrt{ }$ \\
\hline 1 & 1 & 0 & 0 & 1 & $1 \times$ & $1 \sqrt{ }$ \\
\hline 1 & 1 & 1 & 1 & 1 & $1 \sqrt{ }$ & $1 \sqrt{ }$ \\
\hline
\end{tabular}

$$
\text { Sum }=\mathbf{B} \text { and } \mathbf{C}_{\text {out }}=\mathbf{A} \text {. }
$$

The above equation is implemented using a standard Toffoli gate to design the Proposed Adder which is presented in Fig. 3. The Gate level structure of the Proposed Adder is shown in Fig. 4.

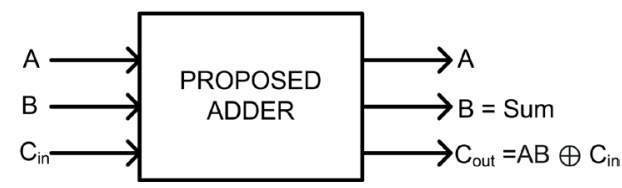

Fig. 3. Proposed Approximate Reversible adder

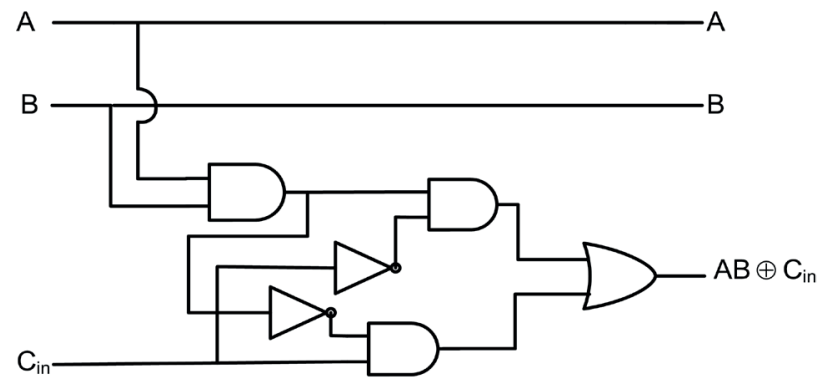

Fig. 4. Gate level Structure of the Proposed Adder

To evaluate the efficacy of the proposed design, we compare the Proposed Adder with reversible full adders and with approximate adders in the literature. These comparison results are presented in Table II. It can be observed that the proposed adder yields better results in terms of power and number of garbage outputs compared to adders existing in the literature.

\section{DESIGN OF NEON USING ThE PROPOSED APPROXIMATE ADDER}

In the previous section, the design of the Proposed Approximate Adder was presented and its efficiency in terms of Power, Delay and Power-Delay Product was compared with the existing design in the literature. In this section, the design of NEON (FIR Filter), which 
TABle II. Performance Evaluation of the Proposed Adder in terms of Power And number of Garbage Outputs

\begin{tabular}{|c|c|c|c|c|}
\hline Design & Reversibility & Technique & Power(nW) & Garbage Outputs \\
\hline \hline Md.Azad khan [30] & $\sqrt{ }$ & Accurate & 4218.991 & 4 \\
\hline HNG Gate [28] & $\sqrt{ }$ & Accurate & 3577.672 & 2 \\
\hline Multiplexer based FA [31] & $\sqrt{ }$ & Accurate & 2513.752 & 3 \\
\hline IC Approx 1 [9] & $\times$ & Approx & 2969.419 & NA \\
\hline IC Approx 2 [9] & $\times$ & Approx & 2432.521 & NA \\
\hline IC Approx 3 [9] & $\times$ & Approx & 1911.338 & NA \\
\hline IC Approx 4 [9] & $\times$ & Approx & 1483.64 & NA \\
\hline XOR/XNOR Approx 1 [10] & $\times$ & Approx & 1460.59 & NA \\
\hline XOR/XNOR Approx 2 [10] & $\times$ & Approx & 2163.708 & NA \\
\hline XOR/XNOR Approx 3 [10] & $\times$ & Approx & 1374.963 & NA \\
\hline Novel Proposed Adder & $\sqrt{ }$ & Approx & $\mathbf{1 1 4 8 . 1 8 6}$ & $\mathbf{1}$ \\
\hline
\end{tabular}

is widely used in many multimedia applications is presented using the Proposed Approximate Adder.

\section{A. Finite Impulse Response (FIR) Filter}

FIR filters are extensively used in many Digital Signal Processing systems intended for multimedia applications. The schematic of a typical FIR filter is presented in this section. An FIR Filter produces a finite impulse response. For a causal discrete-time FIR filter of order ' $\mathrm{N}$ ', each value of the output sequence is a weighted sum of most recent input values. The mathematical representation of a causal discrete-time FIR Filter is presented below.

$$
\begin{aligned}
y[n]=b_{0} x[n]+ & b_{1} x[n-1]+\ldots+b_{n} x[n-N] \\
& =\sum_{i=0}^{N} b_{i} \cdot x[n-i]
\end{aligned}
$$

Where $x[n]$ is the input signal, $y[n]$ is the output signal, ' $\mathrm{N}$ ' is order of the filter and $\mathrm{b}_{i}$ is the value of impulse response at $i^{\text {th }}$ instant for $0 \leq i \leq N$. Input data is multiplied with the filter coefficients and the summation of the results provide the filter response. An FIR filter of 'Nth' order requires ' $N$ ' summations.

In the design of the proposed FIR filter, all conventional adders are replaced by the Proposed Adder cells. FIR filter structures for 8, 12 and 16 orders are designed. The design of an 'N'th order NEON using the Proposed Adder cells is shown in Fig. 5.

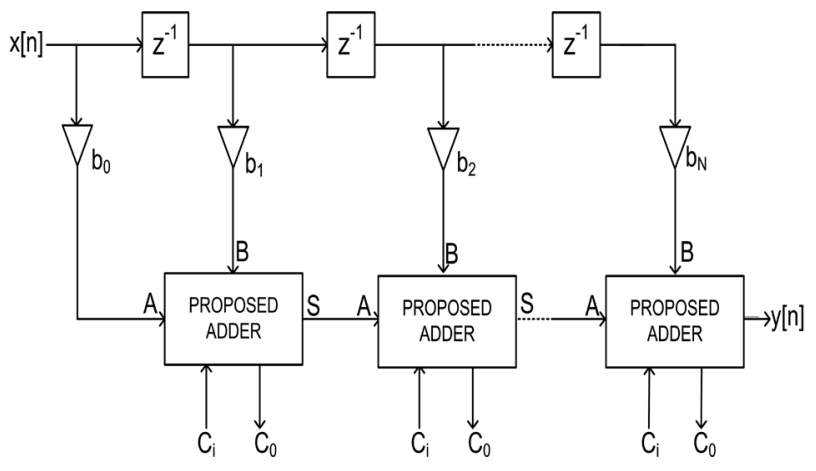

Fig. 5. Design of NEON using the Proposed Adder cells

\section{EXPERIMENTAL RESULTS}

To demonstrate the efficacy of the proposed adder, we have compared its performance with the adders existing in literature [9-10, 30]. The simulation results are carried out using ISCAS Benchmark Suite[35] and the results are very encouraging. These results are tabulated in Table III.

\section{A. Performance Evaluation of NEON}

In this section, we compare the efficiency of 8 , 12 and 16 order NEON with the FIR Filters using existing adders [9, 30-31]. We have embedded each of these adders in designing the FIR Filter (Fig. 5.) and implemented them using Verilog HDL for 8,12 and 16 bit widths of input numbers. We have implemented the aforementioned designs and synthesized them using Cadence design tools at $45 \mathrm{~nm}$ technology node. The performance of NEON in terms of Power, Delay and Power-Delay Product (PDP) is considered with respect to the bit width of the input numbers.

1) Analysis of FIR Filter in terms of Power Dissipation: The performance of NEON in terms of power dissipation for various orders of 8,12 and 16 has been tabulated in Table IV. As the proposed adder produces only one garbage output, the power dissipation of NEON is very much less when compared to FIR filter using existing adders in literature [9, 30-31]. Fig. 6. depicts the average power dissipation measurements of 8,12 and 16 order NEON over the FIR Filter using existing adders[9, 30-31].

TABle IV. Total AVERage Power Dissipation of FIR FILTER OF ORDERS 8,12 AND 16

\begin{tabular}{|c|c|c|c|}
\hline \multirow{2}{*}{ Design } & \multicolumn{3}{|c|}{ Power $(\mathbf{m W})$} \\
\cline { 2 - 4 } & Order 8 & Order 12 & Order 16 \\
\hline Azad Khan[30] & 10.49 & 29.50 & 57.32 \\
\hline Thapliyal[31] & 9.25 & 25.48 & 50.81 \\
\hline Allen [9], Approx4 & 4.31 & 11.37 & 20.66 \\
\hline Proposed Method & $\mathbf{1 . 1 5}$ & $\mathbf{1 . 7 5}$ & $\mathbf{2 . 3 6}$ \\
\hline
\end{tabular}

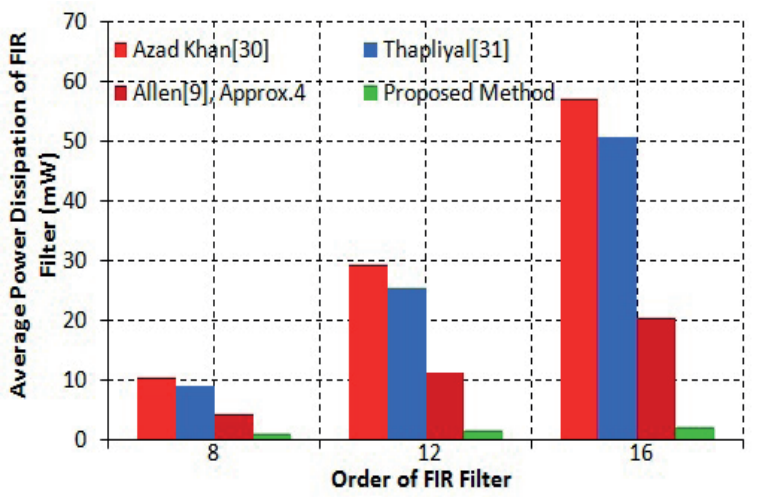

Fig. 6. Total Average Power Dissipation of FIR Filter of orders 8,12 and 16

2) Analysis of FIR Filter in terms of Delay: Table 5 presents the performance of NEON in terms of delay savings for FIR Filters of order 8, 12 and 16. As the design of the Proposed Adder utilizes only a single 
TABlE III. EXPERImEnt Results using ISCAS Benchmarks[35] (POWER(MW), CPU Time(ns), PDP(FJ))

\begin{tabular}{|c|c|c|c|c|c|c|c|c|c|c|c|c|}
\hline \multirow{2}{*}{ Circuit } & \multicolumn{3}{|c|}{ Yang[10],Approx 3 } & \multicolumn{3}{c|}{ Allen[9],Approx 4 } & \multicolumn{3}{c|}{ Azad Khan[30] } & \multicolumn{3}{c|}{ Proposed Method } \\
\cline { 2 - 13 } & Power & CPU Time & PDP & Power & CPU Time & PDP & Power & CPU Time & PDP & Power & CPU Time & PDP \\
\hline c1908 & 84.21 & 62.31 & 5.25 & 85.05 & 62.93 & 5.35 & 85.9 & 63.56 & 5.46 & 32.12 & 22.14 & 0.71 \\
\hline c2670 & 88.42 & 77.26 & 6.38 & 98.14 & 85.76 & 8.42 & 108.94 & 95.19 & 10.37 & 33.21 & 23.89 & 0.79 \\
\hline c5315 & 149.43 & 78.03 & 11.66 & 185.29 & 96.75 & 17.93 & 229.76 & 119.97 & 27.56 & 72.10 & 35.41 & 2.55 \\
\hline c3540 & 170.35 & 104.56 & 17.81 & 223.16 & 136.97 & 30.57 & 292.34 & 179.43 & 52.45 & 81.20 & 39.29 & 3.19 \\
\hline c6288 & 272.56 & 140.12 & 38.19 & 395.21 & 203.17 & 80.29 & 573.05 & 294.59 & 168.81 & 110.32 & 58.42 & 6.44 \\
\hline c7552 & 327.07 & 346.10 & 113.19 & 755.53 & 799.49 & 604.04 & 1745.27 & 1846.82 & 3223.19 & 152.39 & 63.81 & 9.72 \\
\hline s9234 & 346.69 & 508.77 & 176.39 & 429.89 & 630.87 & 271.20 & 533.06 & 782.28 & 417.002 & 154.97 & 78.89 & 12.23 \\
\hline s13207 & 741.93 & 1190.53 & 883.29 & 875.47 & 1404.83 & 1229.89 & 1333.05 & 1657.69 & 1712.47 & 256.39 & 103.92 & 26.64 \\
\hline s15850 & 1187.09 & 1214.34 & 1441.53 & 1424.50 & 1457.21 & 2075.79 & 1709.4 & 1748.65 & 2989.14 & 398.31 & 123.51 & 49.19 \\
\hline s35932 & 1424.51 & 1408.63 & 2006.61 & 1709.41 & 1690.36 & 2889.52 & 2051.28 & 2028.43 & 4160.87 & 412.97 & 169.99 & 70.20 \\
\hline s38417 & 1908.84 & 2366.50 & 4517.27 & 2290.6 & 2839.8 & 6504.85 & 2748.72 & 3407.76 & 9366.97 & 627.14 & 193.21 & 121.17 \\
\hline s38584 3206.86 & 4685.68 & 15026.32 & 3848.16 & 5622.7 & 21637.05 & 4617.79 & 6747.24 & 31157.34 & 721.98 & 202.43 & 146.15 \\
\hline
\end{tabular}

reversible gate, lesser delay is obtained for NEON compared to the FIR filter using existing adders in literature $[9,30-31]$. The delay measurements of 8 , 12 and 16 order NEON over the FIR filters designed using existing adders $[9,30-31]$ is presented in Fig. 7.

TABLE V. DELAY OF FIR FILTER WITH ORDERS 8, 12 AND 16

\begin{tabular}{|c|c|c|c|}
\hline \multirow{2}{*}{ Design } & \multicolumn{3}{|c|}{ Delay (ns) } \\
\cline { 2 - 4 } & Order 8 & Order 12 & Order 16 \\
\hline Azad Khan[30] & 11.00 & 11.41 & 17.37 \\
\hline Thapliyal[31] & 9.93 & 10.16 & 16.47 \\
\hline Allen [9], Approx4 & 6.05 & 8.02 & 10.61 \\
\hline Proposed Method & $\mathbf{2 . 3 8}$ & $\mathbf{2 . 3 9}$ & $\mathbf{2 . 7 8}$ \\
\hline
\end{tabular}

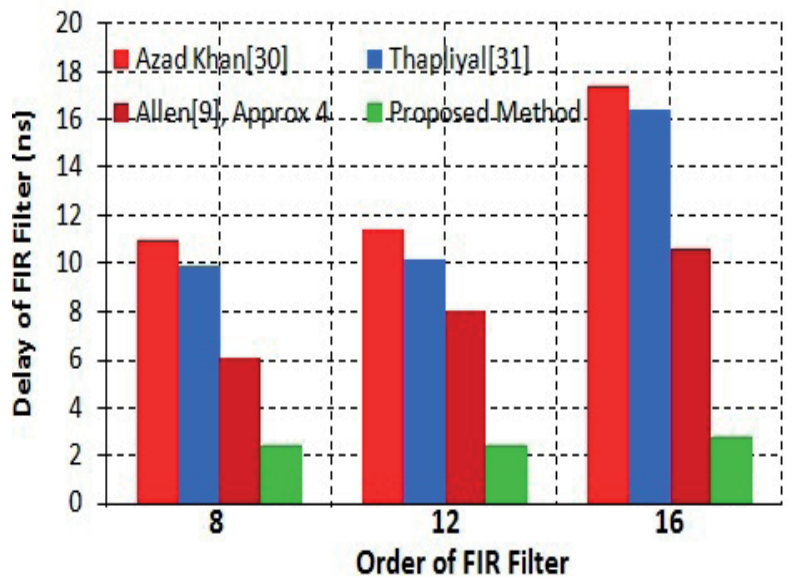

Fig. 7. Delay of FIR Filter of orders 8, 12 and 16

3) Analysis of FIR Filter in terms of Power-Delay Product (PDP): In Table VI, the utility of NEON in terms of Power-Delay Product (PDP) savings is shown. PDP measurement determines the energyefficiency of a system. Savings in terms of Power and Delay of NEON benefits in the reduction of PowerDelay Product as well. Table VI depicts that the PDP of NEON is less compared to FIR filter using adders in [9, 30-31]. PDP measurements of 8, 12 and 16 order NEON over the FIR filter using adders in literature [9, 30-31] is shown in Fig. 8.

TABLE VI. POWER DElay Product of FIR Filter With ORDERS 8, 12 AND 16

\begin{tabular}{|c|c|c|c|}
\hline \multirow{2}{*}{ Design } & \multicolumn{3}{|c|}{ Power Delay Product (pJ) } \\
\cline { 2 - 4 } & Order 8 & Order 12 & Order 16 \\
\hline Azad Khan[30] & 115.39 & 336.59 & 995.64 \\
\hline Thapliyal[31] & 91.85 & 249.70 & 836.84 \\
\hline Allen [9], Approx4 & 26.07 & 91.18 & 218.996 \\
\hline Proposed Method & $\mathbf{2 . 7 3}$ & $\mathbf{4 . 1 8}$ & $\mathbf{6 . 5 6}$ \\
\hline
\end{tabular}

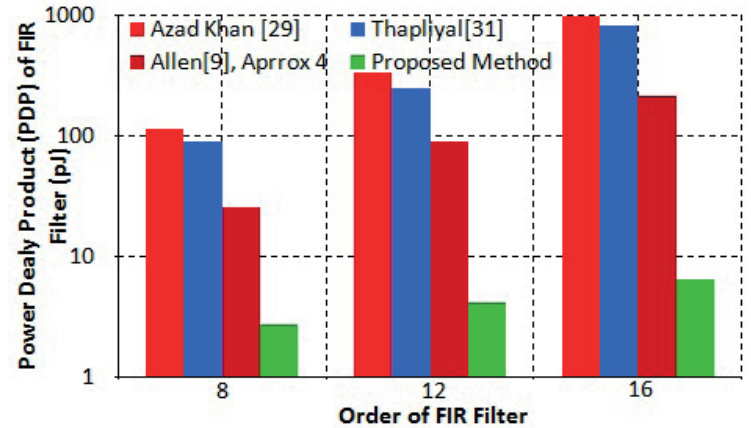

Fig. 8. Power Delay Product of FIR Filter of orders 8, 12 and 16

\section{CONCLUSIONS}

In this paper, we have proposed an Approximate Reversible Adder and analyzed its performance in FIR Filters of various orders. Our approach is aimed at achieving an ultra-low power design of FIR Filter with minimal garbage outputs. We have compared the performance of Proposed Adder in terms of power, delay and PDP with reversible adders and approximate adders in literature. Usage of the Proposed Adder in designing FIR Filters of 8, 12 and 16 orders yielded significant reduction in terms of power, delay and PDP in comparison with the FIR filters designed using adders in the literature.

\section{ACKNOWLEDGEMENT}

This research project was carried out at C-ACRL, Vardhaman College of Engineering. The authors would like to thank the management and faculty for their constant support throughout.

\section{REFERENCES}

[1] Hedge. R, Shanbhag.N, "Energy-efficient signal processing via algorithmic noise-tolerance," in Proc. IEEE/ ACM International Symposium on Low Power Electronics and Design, CA, 1999, pp.30-35

[2] Hedge. R, Shanbhag.N, "Soft Digital signal processing," IEEE Trans. VLSI Syst., vol. 9, no.6, pp.813-823, 2001.

[3] Shim.B, Sridhara.S, and Shanbhag.N, "Reliable low-power digital signal processing via reduced precision redundancy," IEEE Trans. VLSI Syst., vol. 12, no. 5, pp. 497 - 510, 2004.

[4] Varatkar.G and Shanbhag.N, "Energy-efficient motion estimation using error-tolerance," in Proc. IEEE/ ACM International Symposium on Low Power Electronics and Design, 2006, pp. 113 - 118.

[5] Zhu.N, Goh.W.L, and Yeo.K.S, “ An enhanced low-power high-speed adder for error-tolerant application," in Proc. International Symposium on Integrated Circuits, December 2009, pp. $69-72$. 
[6] Chakrapani.L.N, Muntimadugu.K.K, Avinash.L, J.George, and Palem.K.V, "Highly energy and performance efficient embedded computing through approximately correct arithmetic: a mathematical foundation and preliminary experimental validation," in CASES, 2008, pp. 187 - 196.

[7] A. Lingamneni et. al, "Energy parsimonious circuit design through probabilistic pruning," in Design, Automation and Test in Europe Conf and Exhibition (DATE), 2011, pp. 1 6, 2011.

[8] A. Lingamneni et. al, "Algorithmic methodologies for ultraefficient inexact architectures for sustaining technology scaling," in Proc 9th Conf Computing Frontiers, CF'12, (New York, NY, USA), pp. 3 - 12, ACM, 2012.

[9] Allen, C.I., Langley, D.,Lyke, J.C., "Inexact computing with approximate adder application," IEEE National Aerospace and Electronics Conference, NAECON, 2014, pp. 21 - 28.

[10] Zhixi Yang, Jain, A., Jinghang Liang ,Jie Han, Lombardi, F. " Approximate XOR/XNOR-based adders for inexact computing." 13th IEEE Conference on Nanotechnology (IEEENANO), 2013, pp. 690 - 693.

[11] Becker, A. ; Novo, D. ; Ienne, P., "SKETCHILOG: Sketching combinational circuits," Design, Automation and Test in Europe Conference and Exhibition (DATE), 2014, pp. 1-4.

[12] Sung Sik Woo ; Sarpeshkar, R, “ A spiking-neuron collective analog adder with scalable precision," IEEE International Symposium on Circuits and Systems (ISCAS), 2013, pp. 1620 $-1623$.

[13] Landauer.R, "Irreversibility and Heat Generation in the Computational Process", IBM Journal of Research and Development, 5, pp. 183 - 191, 1961

[14] Bennett.C.H, "Logical Reversibility of Computation," IBM J. Research and Development, pp. 525 - 532, November 1973.

[15] Fredkin.E, Toffoli.T, "Conservative Logic," International Journal of Theor. Physics ,21(1982),pp. 219 - 253.

[16] Toffoli.T, "Reversible Computing," Tech memo MIT/LCS/TM-151, MIT Lab for Computer Science (1980).

[17] Feynman.R(1985), Quantum mechanical computers. Optics News, pp. 11 - 20.

[18] Feynman.R(1996), Feynman lectures on computation (A. Hey and R. Allen, eds).Reading: Addison-Wesley.

[19] Thapliyal.H, Srinivas.M.B, "Novel Reversible TSG gate and its application for designing reversible carry look ahead adder and other adder architectures", Proc. of 10th Asia-pacific computer systems architecture Conference, 2005, pp.37-40.

[20] Babu, H.M.H. ; Jamal, L. ; Saleheen, N.,"An efficient approach for designing a reversible fault tolerant n-bit carry look-ahead adder." SOC Conference (SOCC), 2013 IEEE 26th International, 2013, pp. 98 - 103.

[21] Kunalan, D. ; Cheong, C.L. ; Chau, C.F. ; Bin Ghazali, A., “ Design of a 4-bit adder using reversible logic in quantum-dot cellular automata (QCA)," IEEE International Conference on Semiconductor Electronics (ICSE), 2014, pp. 60 - 63.
[22] Lisa, N.J. ; Babu, H.M.H. " Design of a Compact Reversible Carry Look-Ahead Adder Using Dynamic Programming," 28th International Conference on VLSI Design (VLSID), 2015, pp. 238-243.

[23] Bruce.J.W, Thornton.M.A , Shivakumariah.L, Kokate.P.S. and X.Li, "Efficient Adder Circuits Based on a Conservative Reversible Logic Gate," Proceedings of the IEEE Computer Society Annual Symposium on VLSI(ISVLSI'02),Pittsburgh, PA, USA,April 2002, pp 83-88.

[24] Hafiz Md. Hasan Babu, Md. Rafiqul Islam, Syed Mostahed Ali Chowdhury and Ahsan Raja Chowdhury, "Reversible Logic Synthesis for Minimization of Full Adder Circuit," Proceedings of the EuroMicro Symposium on Digital System Design(DSD'03), Belek- Antalya, Turkey,3-5 September 2003, pp-50-54.

[25] Hafiz Md. Hasan Babu, Md. Rafiqul Islam, Syed Mostahed Ali Chowdhury and Ahsan Raja Chowdhury,"Synthesis of Full-Adder Circuit Using Reversible Logic," Proceedings 17th International Conference on VLSI Design (VLSI Design 2004), Mumbai, India,January 2004, pp 757 - 760.

[26] Yvan Van Ientergem and Alexis de Vos, "Optimal Design of A Reversible Full Adder,'Int. Journ. of Unconventional Computing, Vol. 1, pp. 339 - 355.

[27] Haghparast.M and Navi.K, "A Novel Reversible Full Adder Circuit for Nanotechnology Based Systems", J. Applied Sci., pp. 3995 - 4000, 2007.

[28] Haghparast.M and Navi.K, "Design of a Novel Fault Tolerant Reversible Full Adder For Nanotechnology Based Systems," World Appl. Sci. J., pp. 114 - 118 , 2008.

[29] Balasubramanian.P and Mastorakis.N , " High Speed Gate Level Synchronous Full Adder Designs," WSEAS Trans. on Circuits and Systems, Issue 2, Vol. 8,pp 290-300,Feb. 2009.

[30] Md. M. H Azad Khan, "Design of Full-adder With Reversible Gates",International Conference on Computer and Information Technology, Dhaka, Bangladesh, pp. 515-519, 2002.

[31] Thapliyal, H., Srinivas, M.B., "Novel design and reversible logic synthesis of multiplexer based full adder and multipliers", 48th Midwest Symposium on Circuits and Systems, Vol. 2, pp. 1593 - 1596, 2005.

[32] Haghparast.M, Navi.K, "A Novel Reversible BCD Adder For Nanotechnology Based Systems",American Journal of Applied Sciences, Science Publications, pp. 282 - 288, 2008.

[33] Joy.K, Mathew.B.K, "Implementation of a FIR filter model using reversible Fredkin Gate,'IEEE Conference on . ICCICCT, 2014, pp. 690 - 694.

[34] Mishra.B, Al-Hashimi B.M, "Subthreshold FIR Filter Architecture for Ultra Low Power Applications", Proceeding of 18th International Workshop on Power and Timing Modeling, Optimization and Simulation (PATMOS) Lisbon, Portugal, pp.1-10, 2008.

[35] ISCAS Benchmark Suite “ http://www.pld.ttu.eel maksim/benchmarks/iscas89/verilog/" 